\title{
ON NASH'S UNIQUE CONTRIBUTION TO ANALYSIS IN JUST THREE OF HIS PAPERS
}

\author{
SERGIU KLAINERMAN
}

\begin{abstract}
This article focuses on the enormous impact on the modern theory of partial differential equations by three articles of John Nash, all written before the age of 30 , before the onset of his terrible disease.
\end{abstract}

A lot has been written about Nash's legacy in the wake of his tragic death last year. I note in particular Gromov's article [31] on the descendants of Nash's isometric embedding theorems as well as the more comprehensive C. De Lellis survey [18] on the masterpieces of J. Nash. The May 2016 commemorative article in the Notices of American Mathematical Society [19] gives also an excellent account on Nash's work. Rather than repeat what has been said already, I will be short on describing the actual results and concentrate instead on their impact. I will restrict my discussion to only three papers, two of which concern the isometric embedding problem and the third being Nash's work on Hilbert's 19th problem. Both problems are at the center of some of the most exciting, far-reaching developments in PDE and geometry in the last two centuries. All three papers, written within less than ten years from his $\mathrm{PhD}$, had and continue to have a huge impact on geometry and PDEs. At the time when his terrible disease struck, ten years after his PhD, Nash was at the height of his powers, working on fundamental problems in mathematics and physics. One can only imagine how much more he could have achieved!

0.1. Isometric embeddings. The two works [74, [75] on isometric embedding concern a problem first formulated by Schläfli in 1873, whose fundamental character was self-evident in the wake of Gauss and Riemann's foundational works in geometry. As is known, Gauss defined the curvature of a surface as an extrinsic geometric concept and later showed in his Theorema egregium that it is in fact an intrinsic invariant; i.e., it depends only on measurements made on the surface itself, independent of the particular embedding (parametric representation) from which it was first defined. Riemann later took the intrinsic point of view as the starting point for his far-reaching generalization of curvature to higher-dimensional abstract Riemannian manifolds. It was then natural to ask whether these manifolds themselves can be embedded isometrically, i.e., preserving distances and angles, in a finite-dimensional Euclidean space. In modern language the problem amounts to

Received by the editors September 6, 2016.

2010 Mathematics Subject Classification. Primary 35-01.

This article is based primarily on the Nash Memorial Lecture given by the author at Princeton in October 2015. 
finding a smooth $C^{k}$ diffeomorphism 11 large $q$ such that the pull-back of the Euclidean metric $e$ is the given metric $g$ on the manifold $M^{n}$, i.e., $u^{*}(e)=g$. In a local system of coordinates $\left(x^{1}, x^{2}, \ldots, x^{n}\right)$ on $M$ this amounts to

$$
g_{i j}=\partial_{i} u \cdot \partial_{j} u=\sum_{l=1}^{q} \partial_{i} u^{l} \partial_{j} u^{l},
$$

i.e., a fully nonlinear system of partial differential equations (PDE). Note that there are $\frac{n(n+1)}{2}$ independent components of $g$, therefore the system is overdetermined unless $q \geq \frac{n(n+1)}{2}$. Not surprisingly, local, real analytic, isometric embeddings do actually exist $2^{2}$ if $q \geq \frac{n(n+1)}{2}$. The global problem was first tackled in the case when $n=2, q=3$, under positivity assumptions on the Gauss curvature 3 In this case the embedding problem is rigid, i.e., any two solutions differ by a rigid motion in $\mathbb{R}^{3}$.

Theorem (Cohn-Vossen (1927), Herglotz). If $\left(S^{2}, g\right)$ has positive Gauss curvature and $u \in C^{2}\left(S^{2}, \mathbb{R}^{3}\right)$ is an isometric immersion, $\Rightarrow u\left(S^{2}\right)$ is determined up to a rigid motion.

Existence, in this context, was first established by H. Lewy (1937) when the metric $g$ is real analytic, "an analytic metric of positive curvature, defined on a sphere, is always realized by some analytic surface in three-dimensional Euclidean space". It took another twenty years to dispense of analyticity, i.e., to deal with metrics $g$ of limited regularity. The methods introduced to this effect, as developed by Morrey, Nirenberg, Pogorelov, etc., are at the heart of the modern treatment of nonlinear elliptic partial differential equations.

The general problem, as first formulated by Schläfli in 1873, is to find conditions on the dimensions $n, q$ and regularity class $C^{k}$ for which isometric embeddings exist. Challenged by a bet with his MIT colleague W. Ambrose, Nash focused all his impressive intellectual energy on the problem and produced two major breakthrough results. In [74 he established, by a revolutionary new method, the existence of a large class of $C^{1}$ embeddings of compact manifolds $M^{n}$ to $\mathbb{R}^{q}$ for $q \geq n+1$ (in fact, Nash requires $q \geq n+2$; the optimal result was proved by Kuiper [60] a year later by a slight modification of Nash's argument).

Theorem (Nash (1954), Kuiper (1955)). Any short embedding (immersion) $u$ : $M^{n} \longrightarrow \mathbb{R}^{q}, q \geq n+1$ can be uniformly approximated by $C^{1}$ isometric imbeddings (immersions).

\footnotetext{
${ }^{1}$ One can relax this to the requirement that $u$ is an immersion, i.e., with well-defined oneto-one differentials at every point. In that case we talk about isometric immersions instead of embeddings.

${ }^{2}$ This simple application of the Cauchy-Kowalewski principle was first derived by Janet (1926) (see 48]) and Cartan (1927) (see [8]).

${ }^{3}$ The problem was first proposed by H. Weyl in 1916; see 96. Note also that if $C^{2}$ embedding of a $2 D$ compact Riemannian surface in $\mathbb{R}^{3}$ exists, then its Gauss curvature has to be positive at some points.

${ }^{4}$ That is, a $C^{1}$ embedding (immersion) $u$ such that $u^{*}(e) \leq g$ as quadratic forms, or in local coordinates, $\partial_{i} u \cdot \partial_{j} u \leq g_{i j}$. Note that the existence of a global embedding of a general compact manifold $M^{n}$ is by itself a nontrivial problem solved in full generality by Whitney. In general Whitney requires $q \geq 2 n$, but in the case of spheres the condition $q \geq n+1$ suffices.
} 
In [75] Nash proves the more expected version of smooth isometric embeddings from $M^{n}$ to a sufficiently high-dimensional Euclidean space. For $C^{\infty}$ imbeddings the best such result was later proved by Gromov; see [32. More precisely,

Theorem (Nash (1956) [75] and Gromov (1970) [30]). Any short embedding $u: M^{n} \longrightarrow \mathbb{R}^{q}$ can be uniformly approximated by $C^{\infty}$ isometric embeddings (immersions) provided 5 that $q \geq(n+2)(n+3) / 2$.

It is often the case that a new method of proof is more important than the particular result to which it is first applied. In the case of the 1954 result, Nash's new ideas have led to powerful generalizations such as convex integration and the $h$-principle 6 both formulated by Gromov, as well as many applications explained in detail in his survey. The second result is forever tied to the so-called Nash hard implicit function theorem (often called the Nash-Moser implicit function theorem), a stunning, out of the blue, perturbation technique for providing solutions to complicated nonlinear partial differential equations.

0.1.1. Comments on the 1954 theorem. It is incontrovertible that the method of proof of the 1956 theorem is far more important than the particular result it derives 7 The same does not apply to the 1954 theorem. In this latter case the result itself, which uncovers a new phenomenon, is at least as important. It suffices to note that, in the particular case of Weyl's problem, the result seems to be in flagrant contradiction to the Cohn-Vossen rigidity result. Indeed, Nash's result produces highly nonintuitive examples of $C^{1}$ embeddings of the standard sphere of radius 1 in any sphere of radius $R<1$. This highly unexpected result shows, for the first time, that below a certain regularity threshold, solutions to nonlinear partial differential equations 8 may lose their intrinsic predictability (i.e., rigidity in this case). Though, in retrospect, this is undoubtedly the most important message of Nash's work on isometric embeddings, it took many more years before its full meaning

\footnotetext{
${ }^{5} \mathrm{~A}$ further improvement, due to Günther 33 and 34 , requires $q \geq \frac{n(n+1)}{2}+\max \{2 n, 5\}$. On the regularity side the best result, due to Jacobowitz [47], requires metrics of class $C^{2, \alpha}, \alpha>0$. It is not known if the result can be extended to $C^{2}$ metrics; see discussion in 31 and 18 .

${ }^{6} \mathrm{I}$ understand the $h$-principle here, loosely, as high flexibility of the space of solutions to a system of partial differential equations underlying a geometric or a physical problem. This high flexibility is particularly striking, as is the case of Nash's theorem, when it is due to limited regularity rather than indeterminacy of the system. See Gromov [31] and [32] for a precise and more inclusive understanding.

7 The result, at least in so far as it provides existence of smooth embeddings to high-dimensional spaces, was highly expected even though it required an analyst of the class of Nash to deal with the intrinsic difficulties of the problem. In his introduction Nash himself alludes to this when he writes, "The methods used here may prove more fruitful than the results. Time will tell how much can be done with smoothing and feed-back methods."

${ }^{8}$ In his survey [31] Gromov seems to restrict the domain of PDEs to boundary or initial value problems for which uniqueness prevails. Though this is the case for deterministic problems in classical physics, Gromov's definition ignores a vast array of other problems, such as those that appear routinely in quantum physics. Moreover, even when dealing with deterministic problems, the final states of evolution of important nonlinear equations are either stationary or various types of blow-up solutions. These highly nonunique states have to be understood, classified, and connected to the evolution problem at hand. Rather than artificially excluding it from the domain of PDEs, analysts have recognized Nash's discovery as a uniquely important new development and connected it to equations which have nothing to do with the particular geometric problem studied by Nash; see [84, 87, 88, 73], 20, 21], [5], [46], etc. Nash himself makes clear in his introduction in [76] the importance he attributes to the development of new methods in PDE.
} 
was understood and applied to other important nonlinear equations 9 The most far-reaching application of this discovery is in connection to the Onsager conjecture for the incompressible Euler equations.

The Euler equations are a limiting case of the better known Navier-Stokes equations. In the simplest, periodic case, the Navier-Stokes equations describe the evolution of a vectorfield (velocity of fluid particles) $u:[0, T] \times \mathbb{T}^{3} \rightarrow \mathbb{R}^{3}$, in a periodic box $\mathbb{T}^{3}$, i.e.,

$$
\partial_{t} u+u \cdot \nabla u=\nu \Delta u-\nabla p, \quad \operatorname{div} u=0,
$$

where $p=p(t, x)$ denotes the internal pressure and $\nu \geq 0$ the viscosity of the fluid. The Euler equations are obtained by taking $\nu=0$. One is interested in Euler flows $u=u(t, x)$ with prescribed initial condition at $t=0$. It can be easily shown that $C^{1}$ solutions with the same initial data are unique, i.e., any two $C^{1}$ solutions which coincide at a given time must coincide for all time, and conserve the energy integral $E(t)=\int_{\mathbb{T}^{3}}|u(t, x)|^{2} d x$, i.e., $E(t)=E(0)$ for all $t$. The Onsager conjecture, which has its origins in Kolmogorov's theory of turbulence, concerns the question of the minimal regularity needed for weak solution 10 of the equations to conserve the energy. The simplest way to measure regularity (in a continuous fashion) is with respect to Hölder spaces $C^{\alpha}$. We say that a weak solution $u$ belongs to $L_{t}^{\infty} C^{\alpha}$ if there exists a constant $C>0$ such that

$$
\left|u\left(t, x_{1}\right)-u\left(t, x_{2}\right)\right| \leq C\left|x_{1}-x_{2}\right|^{\alpha}, \quad \forall t \in \mathbb{R}, x_{1}, x_{2} \in \mathbb{T}^{3} .
$$

Conjecture (Onsager 82 (1949)). Weak $L_{t}^{\infty} C^{\alpha}$ solutions of the Euler equations conserve the energy $E$ if $\alpha>1 / 3$. If $\alpha<1 / 3$, there exists weak solutions which do not preserve the energy.

The Onsager $1 / 3$ exponent is intimately connected to the power spectrum predicted by Kolmogorov [56] in connection with his turbulence theory for the $3 D$ Navier-Stokes. The conjecture itself was motivated by Onsager's belief that anomalous dissipation of energy for Navier-Stokes may be explained by the behavior of solutions to the Euler equations (see [2, 20], 29], 92] for recent reviews on the Onsager conjecture and its relations to turbulence). The positive part of the conjecture was first addressed in [28, and settled 11 in 9 .

Remarkably, one can interpret the second, harder, part of the conjecture in the context of the $h$-principle and convex integration discovered by Nash for the isometric embedding problem. Though the formal connection between Nash's result and the Onsager conjecture was first made by C. De Lellis and L. Székelyhidi Jr. in [21, constructions of specific weak, highly nonunique, solutions to the Euler equations were made already in 84, 87, and 88. Starting with the breakthrough work of De Lellis and Székelyhidi [21], which constructs anomalous weak $L^{\infty}$ solutions by convex integration, progress on the second part of the Onsager conjecture has been extraordinarily fast. In another major breakthrough they were able to adapt

\footnotetext{
${ }^{9}$ It is interesting to note in this regard that Nash himself did not seem to think very highly of his $C^{1}$ result, which he viewed as a sidetrack by comparison to the smooth embedding result (see his own comments in the Raussen-Skau interview in [19]).

${ }^{10}$ These are solutions defined in a distributional sense. One can make sense in this way of solutions which are merely in $L^{2}\left(\mathbb{R} \times \mathbb{T}^{3}\right)$.

${ }^{11}$ The sharpest result available in 11] shows the energy conservation holds true for flows in the closure of $C_{c}^{\infty}$ in $L_{t}^{3} B_{3, \infty}^{1 / 3}$, with $B_{3, \infty}^{1 / 3}$ a Besov space slightly larger than the more familiar $L^{3}$ based Sobolev space with $1 / 3$ derivatives.
} 
the method of convex integration to construct continuous solutions which dissipate energy (see 22]) and, by tweaking the result a bit, to go up to Hölder exponents $\alpha<1 / 10$ (see [23]). The result was then improved by Isett to $\alpha<1 / 5$ (see [44] and the shorter proof in [5]). Further progress has been made in [6] where the authors show that $h$-principles are available for solutions that are integrable in time with values in a Holder space, i.e., $L_{t}^{1}\left(C^{\alpha}\right)$ with $\alpha<1 / 3$. Moreover, in [7] it has been very recently proved that the $h$-principle holds for weak solutions in $L_{t}^{\infty}\left(H^{1 / 3-}\right)$ (i.e., with respect to the $L^{2}$ based Sobolev spaces $H^{s}, s<\frac{1}{3}$ ) thus matching the Kolmogorov 5/3-power spectrum. Finally, just as I was writing this part of my essay, I have received a manuscript from P. Isett with a full proof of the Onsager conjecture in $L_{t}^{\infty} C^{\alpha}, \alpha<\frac{1}{3}$; see [45]. The final proof, based on a vast extension of the convex integration ideas of Nash, gives a perfect illustration of how important ideas to solve one specific PDE may have an impact in a completely unrelated one.

Besides the incompressible Euler equations, convex integration methods have been successfully used in fluid dynamics to establish the nonuniqueness of weak solutions below a certain rigidity threshold; see for example the review article [20]. Remarkably, most of these results concern bounded weak solutions, i.e., solutions in $L_{t}^{\infty} L_{x}^{\infty}$ and encounter a fundamental difficulty (due to high frequency interactions) in obtaining solutions which are continuous in space. Note that in the case of the Euler equations the passage from merely bounded to continuous in space, made originally by De Lellis and Székelyhidi in [22, was made with the help of a special class of stationary solutions of the Euler equation, i.e., Beltrami flows. In his recent solution of the Onsager conjecture, Isett uses another kind of stationary flows, i.e., Mikado flows 12 A different type of additional structure was found 13 for the incompressible porous medium equation, or more generally incompressible active scalar equations with nondegenerate 14 nonodd constitutive laws by P. Isett and V. Vicol (see [46]) but it is not yet known for other physically important equations, such as the compressible Euler equations of isentropic gas dynamics. Nonuniqueness and $h$-principles in this case were established for the class of $L_{t, x}^{\infty}$ weak solutions [12, 24, 64. It remains currently an open problem as to whether $h$-principles hold for a smoother class of weak solutions.

The work of De Lellis and Székelyhidi has opened the way not only for a better understanding of the Onsager conjecture and related results for other equations in hydrodynamics but has also led to a better appreciation of the importance of understanding the transition, in the context of the isometric embedding problem, between the regime of strong nonuniqueness discovered by Nash to that of rigidity, as in the Cohn-Vossen theorem. As in the case of the Onsager conjecture, it is tempting to believe that a sharp Hölder regularity transition exponent exists in the particular case of embedding $2 D$ Riemannian metrics to $\mathbb{R}^{3}$. It is known (see [25]

\footnotetext{
${ }^{12}$ In the context of convex integration for the Euler equations these were first used in [26].

${ }^{13}$ The first $h$-principle type result for these equations were first shown to hold for $L^{\infty}$ weak solutions in 10 89 93 . Using the specific structure of incompressible transport, a serial convex integration scheme (in the spirit of the scheme of Nash for the isometric embedding), Isett and Vicol were able to obtain $h$-principles for Hölder continuous weak solutions with exponent < $1 /(4 d+1)$ (here $d$ denotes the space dimension).

${ }^{14}$ They also show that the $h$-principle fails if the nondegeneracy condition is not verified.
} 
and the reference within) that the critical exponent, if it exists, must lie 15 between $C^{1,1 / 5}$ and $C^{1,2 / 3}$; see 14 and [18 for an up to date account of the state of the problem.

0.1.2. Comments on the 1956 theorem. As mentioned earlier, the most important thing about Nash's 1956 paper is the introduction of a revolutionary new method to construct smooth local solutions to complicated nonlinear partial differential equations, in a neighborhood of a given solution, by an iterative process which allows loss of derivatives. To be more precise, one of the main steps in his 1956 paper is to construct solutions to nonlinear equation (0.1) close, in some sense, to a given solution. Thus, if we denote by $F$ the functional $F(u)=\left[\partial_{i} u \cdot \partial_{j} u\right]_{i, j=1, \ldots, n}$ and consider a given solution of (0.1) $F(u)=g$, then the goal is to solve the system

$$
F(u+v)=g+f
$$

for small perturbations $v$ and $f$. Taking the formal Fréchet derivative of $F$, one derives the linearized system $F^{\prime}(u) v=h$ or, in coordinates,

$$
\partial_{i} u \cdot \partial_{j} v+\partial_{j} u \cdot \partial_{i} v=h_{i j},
$$

which is a system of $\frac{n(n+1)}{2}$ equations for $q$ variables. To solve this complicated linear system, Nash's trick is to add equations, i.e.,

$$
v \cdot \partial_{i} u=0 \text {. }
$$

Differentiating (0.5) and combining with (0.4) leads to the simpler linear system

$$
\partial_{i} \partial_{j} u \cdot v=-\frac{1}{2} h_{i j}, \quad v \cdot \partial_{i} u=0,
$$

which can be easily solved provided that $\partial_{i} u, \partial_{i} \partial_{j} u, i, j=1, \ldots, n$, are linearly independent. This, of course, implies $q \geq \frac{n(n+1)}{2}+n=\frac{n(n+3)}{2}$. The problem is that if $u \in C^{k}$, then the solution $v$ of (0.6) can only by in $C^{k-2}$, i.e., we lose two derivatives. Any reasonable person would conclude that this procedure would lead nowhere, in the smooth category 16 since any iterative process based on this linearization would have to lose infinitely many derivatives. But not Nash! His inner voice must have been telling him to persist where others would have given up. That led him to a revolutionary new method to deal with loss of derivatives.

Despite appearances $\sqrt{17}$ it is not too difficult to describe the main ingredients of the new method; see Appendix A

0.1.3. Impact of Nash's iteration technique. Reading the introduction to his 1958 Annals paper [76] (as well as his remarks in the introduction to 75], see footnote 7), it is clear that Nash was keenly aware of the possibility to apply his scheme to other problems in nonlinear PDEs:

\footnotetext{
${ }^{15}$ In 25 the authors show that the $h$-principle holds for isometric $C^{1,1 / 5-\delta}$ immersions of twodimensional disks, endowed with $C^{2}$ Riemannian metrics, in $\mathbb{R}^{3}$. The rigidity result for $C^{1,2 / 3+\delta}$ maps is due to Borisov, with a shorter proof in 14 .

${ }^{16}$ Cauchy-Kowalewsky allows one to overcome loss of derivatives in the analytic case.

${ }^{17}$ In his essay, Gromov is right to criticize various attempts to codify Nash's scheme into a general, precise theorem which can then be applied to a vast range of problems. It is better, in my experience, to understand Nash's technique in the simplest possible setting and, if needed, adapt it to any new situation at hand.
} 
The open problems in the area of nonlinear partial differential equations are very relevant to applied mathematics and sciences as a whole, perhaps more so than the open problems in any other area of mathematics, and this field seems poised for rapid development. It seems clear, however, that fresh methods must be employed. We hope this paper contributes significantly in this way and also that the new methods used in our previous paper [75, will be of value.

The first attempts to codify Nash's method into a new type of implicit function theorem were made by J. Schwartz [85] and, more successfully, by J. Moser 68], [69]. Moser was in fact so successful in simplifying Nash's technique, using a Newton iteration, that the method was later universally referred to as Nash-Moser. Moser was also the first to find an application of his simplified scheme to small divisor problems in celestial mechanics. Another comprehensive study of the Nash-Moser technique was later made by R. Hamilton in [35] in which the very useful concept of "tame maps" is developed and applied to a $C^{\infty}$ version of the Nash-Moser hard implicit function theorem. The new method is later revisited by Hörmander [43] who pioneers a discretized version of the original Nash scheme. As pointed out in [43, the original Nash scheme produces better results than the Nash-Moser scheme 18

Important applications started to pour in during what one might call the NashMoser expansion period (1962-1982). Here are just a few examples:

(1) L. Nirenberg (1972) formulates an abstract, nonlinear Cauchy-Kowalewski theorem using Nash-Moser; see [79.

(2) F. Sergeraert (1972) is first to give a $C^{\infty}$ version of the Nash-Moser theorem, using the framework of Fréchet spaces with some applications to small divisor problems; see 86 .

(3) M. Kuranishi (1975) applies the Nash-Moser technique for deformations of isolated singularities in the context of the $\bar{\partial}_{b}$ in complex analysis; see [61.

(4) D. Schaeffer (1975) finds simple applications of the technique to free boundary value problems; see 83 .

(5) L. Hörmander (1976-1977) applies the technique to the geodesy problem; see 42. He also revives the original Nash iteration technique by discretizing and presenting it in the framework of tameness introduced by Hamilton; see 43 .

(6) S. Klainerman (1980) uses the technique to prove the first global existence results for a broad class of nonlinear wave equations; see [49. The novelty in this case was loss not of derivatives but rather los 19 of decay.

(7) R. Hamilton (1982) gives a very elegant and comprehensive treatment of the Nash-Moser technique in 35 and applies it to his recently introduced Ricci flow; see 36.

(8) S. Alinhac (1995) uses the Nash-Moser technique to prove novel blow-up results for quasilinear wave equations in higher dimensions; see 1 .

(9) H. Lindblad (2005) applies Nash-Moser to water wave problems; see 63.

(10) M. Clément and C. Villani (2011) use the Nash-Moser technique in their recent groundbreaking work on Landau damping; see [13.

\footnotetext{
${ }^{18}$ This fact played an important role in my own work on nonlinear wave equations 49.

${ }^{19}$ Solutions of the linear wave equation decay like $t^{-\frac{n-1}{2}}$ in the uniform norm. The original scheme used in 49] was losing one of order of decay for every iterate.
} 
(11) P. Hintz and A. Vasy (2016) have recently proved the global nonlinear stability of the small angular momentum Kerr-De Sitter black holes using the Nash-Moser technique; see [41.

The euphoria, produced by the realization that many difficulties previously considered impossible can be resolved by the new method, lasted roughly until the 1980 s, when analysts slowly started to discover classical proofs of the new results. Thus, for example, the apparent loss of derivatives of the Ricci flow, which led Hamilton to use Nash-Moser, could be overcome by a judicious choice of a gauge condition 20 see DeTurck [17]. The loss of decay manifest in [49] could be overcome by better linear estimates; see [89], [54, and [50. Most surprisingly, the loss of derivatives in the isometric embedding problem itself was overcome in 1989 by Günther [33, 34] by a clever use of nonlocal, pseudo-differential operators. All other uses of Nash-Moser mentioned in the list above were shown later to be unnecessary 21 (to my knowledge the only type of results which still require Nash-Moser are connected to small divisor problems, such as in smooth KAM theory). Today the Nash-type iteration should be viewed as a technique of last resort: if nothing else works, try Nash-Moser! Though most proofs based on Nash-Moser were later replaced by classical ones by people who dig deeper into the problem at hand, the technique continues to have great conceptual and psychological value. As such, it is important for analysts to recognize the features of a linearized problem, with apparent loss of derivatives, which can be treated by the Nash-Moser techniques; see Appendix $\mathrm{A}$ for a very quick introduction and [43] for a full exposition.

0.1.4. Open problems in connection to the isometric embedding papers. In his survey [31. Gromov gives an impressive list of 85 open problems in geometry directly connected to the work of Nash on isometric embedding. My personal view is that the most important discoveries made by Nash resonate way beyond the isometric embedding context to the broader field on nonlinear PDEs. It was widely believed, Nash himself included, that the most important applications of the isometric embedding papers are related to the hard implicit function methodology developed in [75. The first to draw attention to the revolutionary potential of the $C^{1}$ embedding result was Gromov who distilled from it his general $h$-principle and the method of convex integration. The $h$-principle is particularly interesting in problems where, above a certain regularity threshold, one has rigidity. This applies, most importantly, in my view, to the initial value problem for nonlinear, Hamiltonian PDEs with a well defined conserved, coercive, energy integral. In each of these equations there are various important regularity thresholds, similar to the one encountered for the Euler equation. Without being specific, let us denote by $a_{U}$ and $a_{O}$ the regularity thresholds corresponding to uniqueness and conservation of energy for the particular problem at hand. In other words, $a_{U}$ measures the space-regularity of solutions, with respect to a given continuous, ordered set of norms indexed by $a$ (with a corresponding family of Banach space $B_{a}$ ), such that uniqueness prevails above $a_{U}$ and fails below $a_{U}$. Similarly $a_{O}$, just as in the case of the Onsager conjecture, denotes the regularity threshold for which conservation of energy holds

\footnotetext{
${ }^{20}$ Remarkably, the DeTurck trick was actually known in the general relativity community, as it was used already in the famous Y. C. Bruhat [3] local-in-time existence and uniqueness result for the Einstein equations.

${ }^{21}$ The work of Hintz and Vasy is too new, yet I am certain that Nash-Moser is also unnecessary in that context.
} 
true. Finally, we can define $a_{N}$ as the optimal regularity threshold below which some version of the Nash's $h$-principle holds true for solutions of the initial value problem. Note that both $a_{N}$ and $a_{U}$ were relevant to the isometric embedding works of Nash, the first connected to the $C^{1}$ embedding, the second to the higher regularity result in 75 . No analogue of the Onsager exponent $a_{O}$ is known in that case, however, since there is no analogue of energy conservation.

Another important regularity threshold is $a_{W P}$, the well-posedness exponent above which one can prove local existence and unique dependence of the data in the corresponding family of Banach spaces $B_{a}$. We have a very general result, which applies to large classes of equations, which establishes well-posedness for sufficiently large $a$. In the case of the incompressible Euler equation, the best well-posedness result 22 is $C^{1, \alpha}, \alpha>0$, in Hölder spaces and $H^{a}, a>n / 2+1$, in Sobolev spaces $H^{a}$, where $n$ is the space dimension. Both bounds are sharp, in view of a result of Bourgain and Li; see 4. Typically, for most other important Hamiltonian equations, only the $L^{2}$ Sobolev spaces $H^{a}$ can be used to establish well-posedness 23 In most interesting cases, as it turns out, the spaces $B_{a}, a \geq a_{W P}$, are much smaller than the energy space $E$. These equations are called supercritical, and they play a fundamental role in the nonlinear PDE world; see [51] for a more comprehensive discussion.

Typically 24 it is difficult to find the correct value of $a_{W P}$. We have, however, a more informal way to define supercriticality by using the so-called scaling exponent $a_{*}$ of the given equation. This $a_{*}$ is the precise value of $a$ for which the corresponding homogeneous Banach space $25 \dot{B}_{a}$ is a scale-invariant relative to simple scale transformations which preserve the space of solutions of the equations. In the case of the Euler equations, relative to the standard space-time scaling (i.e., $\left.u_{\lambda}(t, x)=u(\lambda t, \lambda x), p_{\lambda}(t, x)=p(\lambda t, \lambda x)\right)$, we have $a_{*}=0$ in Hölder spaces and $a_{*}=\frac{n}{p}$ in $W_{p}^{a}$ spaces. By simple scaling considerations one can show, in general, that $a_{*} \leq a_{W P}$. A problem may thus be called supercritical, or strictly supercritical, if $B_{a_{*}}$ is strictly smaller than the energy space $E$; see [51] for a discussion of scaling and scaling exponent and relation to well-posedness. The classical well-posedness results, alluded to before, establish well-posedeness for exponents $a>a_{*}+1$. The Bourgain-Li result [4] shows that in fact $a_{W P}=a_{*}+1$ for Euler, which illustrates the degenerate nature of the equations. For nonlinear wave equations one can show that the gap is far smaller. In fact some of the deepest recent results for semilinear geometric wave equations, establish some version of well-posedness for all $a \geq a_{*}$, in the framework of Sobolev spaces $H^{a}$, for realistic dimensions. These results played a fundamental role in establishing large data global regularity results in critical dimensions 26 In the case of quasilinear equations, such as the Einstein field equation in three space dimensions, it is known that well-posedness holds for $a \geq a_{*}+\frac{1}{2}$ (see

\footnotetext{
${ }^{22}$ Well-posedeness also holds in $W_{p}^{a}$ spaces for $a>n / p+1$. This result is also sharp.

${ }^{23}$ This is particularly the case of nonlinear systems of hyperbolic equations in space dimensions $n>1$. The incompressible Euler equations can be viewed as a degenerate case of a hyperbolic system, with no dispersion.

${ }^{24}$ The incompressible Euler equation is an exception in this regard.

${ }^{25}$ The subspaces of $B$ which have a well-defined scale. In the context of Sobolev spaces these are the usual dot spaces $\dot{W}^{s, p}$ spaces.

${ }^{26}$ See [90,91], [58], and 94 95] for wave maps, 80 81] for Maxwell-Klein-Gordon, and [57] for Yang-Mills, as well as the references within.
} 
[55]), and it is a major open problem whether one can bridge the gap, or even do slightly better.

The presence of these five critical exponents in a given system raise the following natural questions.

Given an (important) nonlinear evolution, supercritical, Hamiltonian system, does there there exist an h-principle?

What are the critical exponents $a_{U}, a_{N}, a_{O}, a_{W P}$, and what are the relations between them?

How do the corresponding spaces $B_{a}$ compare to the energy space $E$ and scale invariant space $B_{a_{*}}$, and what do these spaces have to say about the general evolution of the system or its connection to related ones?27

Are there other critical exponents still to be discovered?

So far, versions of the $h$-principle have been found only for hydrodynamics equations, yet we do not have any reason to exclude the possibility that similar phenomena occur for other important PDEs such as the Einstein field equations or various other examples of quasilinear geometric wave equations. It is interesting to note that, in the familiar regime of Hölder spaces, for the Euler equations we have 28

$$
0=a_{*}<a_{O} \leq \cdots a_{N} \cdots \leq a_{U} \leq a_{W P}=1 .
$$

Is it conceivable that such a relation holds more generally? Note that if an $h$-principle holds and $a_{*}<a_{N}$, then, in particular, there must be a gap between $a_{*}$ and $a_{U}$ and therefore also between $a_{*}$ and $a_{W P}$. Thus some version of the $h$-principle could be used, in principle, to demostrate the existence of such a gap for the Einstein field equation, 29 or other geometric quasilinear systems of wave equations.

In the context of the Euler equations the most important open problem, concerning the above exponents, are the precise values of $a_{U}$ and $a_{N}$. So far we know that uniqueness holds for $C^{1}$ flows, but there is no known reason why one could not go lower. Can one extend convex integration methods to construct solutions above $a_{O}$ (i.e., nonunique weak solutions which conserve the energy but fail to conseve other known, higher-order quantities such as helicity)?

An even more fundamental problem, left open in the wake of remarkable recent full resolution of the Onsager conjecture by P. Isett, is to connect the convex integration methods developed in the inviscid case to turbulent flows for Navier-Stokes equations, that is, to justify fully the original insight of Onsager 30 An obvious limitation of convex integration, as known today, is that it produces too many solutions of the Euler equations, in particular solutions which correspond to arbitrary energy profiles, while vanishing viscosity limits of solutions to the Navier-Stokes equations cannot increase the energy.

\footnotetext{
${ }^{27}$ Such as the relation between Euler and Navier-Stokes. The Onsager conjecture itself was inspired by considerations concerning anomalous energy dissipation for Navier-Stokes.

${ }^{28}$ We do not know the precise values of $a_{N}$ and $a_{U}$, but it is reasonable to assume that $a_{O}<a_{U}$. Can $a_{N}$ be equal to $a_{U}$ ?

${ }^{29}$ The scaling exponent, in $H^{a}$-Sobolev spaces, is $a_{*}=3 / 2$ while, as mentioned above, the best known well-posedness result in [55] shows that well-posedness holds for $a \leq 2$. It is a major open question in the subject whether $a_{W P}=a_{*}$ or even whether one can go below 2 .

${ }^{30}$ Concerning the Kolmogorov power spectra and the $4 / 5$ law, in the context of the Euler equations; see also the recent treatment in [7].
} 
0.2. Hilbert's 19th problem. Both the pedigree and impact of Hilbert's 19th problem are even more impressive than those of the isometric embedding. If the latter had its source in the revolutionary works on curved spaces by Gauss and Riemann, the former is tied to the variational principle, or the principle of least action as it is better known in physics. The principle, which has its origin in the insights made by mathematicians such as Fermat, Leibniz, Maupertius, the Bernoulli brothers, Euler, Lagrange, Hamilton, and E. Noether in connection to geometric and physical problems, is now both a cornerstone of modern physics as well as a powerful method to generate rich geometric objects, such as minimal surfaces, harmonic maps, Yang-Mills connections, etc.

It was Riemann who first attempted to use the variational principle (more precisely the Dirichlet principle) to prove a mathematical result, the well known Riemann mapping theorem. Riemann's proof, which was found to be seriously defective by Weierstrass, was corrected many years later by Hilbert. Hilbert's novel functional set-up to formulate and prove the Dirichlet principle opened the way for attacking other important variational problems and is now a foundational stone of the modern theory of elliptic PDEs. In his address to the 1900 International Congress of Mathematicians, Hilbert stated his 19th problem as follows (see [40]): "Does every Lagrangian partial differential equation of a regular variation problem have the property of admitting analytic integrals exclusively?" In modern, more genera 31 language the problem refers to second order, scalar, elliptic PDEs which arise variationally from real analytic Lagrangians, $L(p, z, x)$, with $p=\left(p_{1}, \ldots, p_{n}\right) \in \mathbb{R}^{n}$, $z \in \mathbb{R}, x=\left(x^{1}, \ldots, x^{n}\right) \in \mathbb{R}^{n}$, convex relative to the variables $p$; i.e.,

$$
\sum_{i, j=1}^{n} \partial_{p_{i}} \partial_{p_{j}} L(p, z, u) \xi_{i} \xi_{j}>0, \quad \forall \xi \in \mathbb{R}^{n} .
$$

Hilbert's problem is to show that the minimizers of the action integral $\mathcal{I}[u]=$ $\int_{D} L[\nabla u, u, x] d x$ for functions $u: D \subset \mathbb{R}^{n} \longrightarrow \mathbb{R},\left(\nabla u=\left(\partial_{1} u, \ldots, \partial_{n} u\right)\right.$ the gradient of $u$ in $x)$ with appropriate boundary conditions at $\partial D$, are necessarily real analytic, or just infinitely differentiable 32 in $D$. If such a minimizer $u=u(x)$ exists, it must satisfy, of course, the second order PDE,

$$
\sum_{i=1}^{n} \partial_{i} \partial_{p_{i}} L(\nabla u, u, x)=\partial_{z} L(\nabla u, u, x), \quad \partial_{i}:=\frac{\partial}{\partial x^{i}},
$$

called the Euler-Lagrange equations. The main examples Hilbert had in mind were the Dirichlet Lagrangian $L=|p|^{2}$, corresponding to the Dirichlet functional $\mathcal{I}[u]=\int_{D}|\nabla u|^{2} d x$, and $L=\left(1+|p|^{2}\right)^{1 / 2}$ corresponding to the functional $\int_{D}\left(1+|\nabla u|^{2}\right)^{1 / 2} d x$, for surfaces given by the graph of scalar function $u$. The EulerLagrange equation of the first is the Laplace equation while that of the second is the minimal surfaces equation.

The solution to the problem also has a venerable history. It was Bernstein in 1904 and later Petrowski, E. Hopf, J. Schauder, C. B. Morrey, and L. Nirenberg who were able to show that sufficiently regular solutions of the corresponding Euler-Lagrange equation must be infinitely differentiable or analytic. On the other hand, the direct variational methods pioneered by Riemann and Hilbert were able to produce weak

\footnotetext{
${ }^{31}$ Hilbert formulates his problem in two dimension, i.e., $n=2$.

${ }^{32}$ Though Hilbert requires analyticity, it turns out that the main difficulty is to show that the solutions are sufficiently smooth.
} 
solutions of the Euler-Lagrange equations. More precisely, in the particular case when $L=L(p)$ and

$$
c^{-1}|\xi|^{2} \leq \sum_{i, j=1}^{n} \partial_{p_{i}} \partial_{p_{j}} L(p) \xi_{i} \xi_{j} \leq c|\xi|^{2}, \quad \text { for some } \quad c>0,
$$

a unique minimizer can be shown to exist in the Sobolev space $H^{1}$ (i.e., $\nabla u \in L^{2}$ ) which verifies the Euler-Lagrange equations,

$$
\sum_{i=1}^{n} \partial_{i}\left(\partial_{p_{i}} L(\nabla u)\right)=0
$$

By a simple calculation one can show that each derivative $w=\partial_{i} u \in L^{2}$ verifies

$$
\sum_{i, j=1}^{n} \partial_{i}\left(C^{i j}(x) \partial_{j} w\right)=0, \quad C^{i j}(x):=\partial_{p_{i}} \partial_{p_{j}} L\left(\nabla_{x} u(x)\right) .
$$

We are thus led to study linear second order equations with measurable coefficients $C^{i j}$ for which we know nothing more than the uniform ellipticity estimate derived from (0.8),

$$
c^{-1}|\xi|^{2} \leq C^{i j}(x) \xi_{i} \xi_{j} \leq c|\xi|^{2} .
$$

To succeed in proving Hilbert's generalized 19th problem, in any dimension $n$, one has to show that any $L^{2}$ solution $w$ of the linear equation (0.9), with barely measurable coefficients $C^{i j}$ verifying (0.10), must be sufficiently differentiable so that the previous methods, pioneered by Bernstein, Petrowsky, E. Hopf, J. Schauder, etc., apply. To bridge the gap, it suffices in fact to show that $w$ is slightly better than continuous, i.e., Hölder continuous $C^{\epsilon}$ for some exponent $\epsilon>0$. This step was first accomplished in a historic paper by Morrey [66 in 1938 in the particular case of two space dimensions, the original version of Hilbert's problem. The methods used by Morrey, based on complex analysis and quasiconformal mappings, were heavily restricted to two dimensions. Morrey also understood (see [67]) that the key to extending his results to higher dimensions was to prove the above-mentioned Hölder estimate for $w$.

This state of affairs in the early 1950s opened the way for two brilliant young mathematicians, E. DeGiorgi and J. Nash, to make one of the most important contribution, to mathematics in the second half of the twentieth century. The story is well known; see S. Nassar [77. Nash, who loved to probe mathematicians he trusted about the most important open problems in their fields, got interested in this Hölder regularity issue via L. Nirenberg. Though completely unversed in the subject 33 Nash succeeded in solving the problem by a characteristic display of originality. In fact, he not only solved the original problem but a seemingly harder one- that is the parabolic problem

$$
\partial_{i}\left(C^{i j}(x, t) \partial_{j} w\right)=\partial_{t} w
$$

for which he proved the following result.

\footnotetext{
${ }^{33}$ According to L. Nirenberg, at the very beginning of his interest in the problem Nash would come to him every two weeks or so with very naive questions. Nirenberg was later both surprised and awed by Nash's solution.
} 
Theorem (Nash (1958) [76]). Assume that the coefficients $C^{i j}(t, x)$ verify (0.10) and that $|w(x, t)| \leq B$ for all $t \geq t_{0}$, then 34

$$
\left|w\left(x_{1}, t\right)-w\left(x_{2}, t\right)\right| \leq B A\left(\frac{\left|x_{1}-x_{2}\right|}{\left(t-t_{0}\right)^{1 / 2}}\right)^{\alpha}
$$

with $A>0$ and $\alpha>0$ depending only on the uniform ellipticity constant $c$ in (0.10) and dimension $n$. As a corollary, the same result holds true for the elliptic problem (0.9).

The introduction to [76] gives the impression that Nash regarded this result as his most important to date. Indeed, reading his unusually long (for him) "general remarks", one has the impression that he had high expectations that his new a priori estimates could open the way to make progress on one of the most fundamental problems in hydrodynamics, indeed of all of science - the problem of turbulence.

Little is known about the existence, uniqueness and smoothness of solutions of the general equations of flow for a viscous, compressible and heat conducting fluid. These are a non-linear parabolic system of equations. An interest in these questions led us to undertake this work. It became clear that nothing could be done about the continuum description of general fluid flow without the ability to handle non-linear parabolic equations and that this in turn required an a priori estimate of continuity such as (0.12). Probably one should first try to prove a conditional existence and uniqueness theorem for the flow equations. This should give existence, smoothness, and unique continuation (in time) of flows, conditional on the non-appearance of certain gross types of singularity, such as infinities of temperature or density. (A gross singularity could arise, for example, from a converging spherical shock wave.) A result of this kind would clarify the turbulence problem.

Given the importance Nash attributed to this work, it is not hard to understand his great disappointment when he found out that a similar result was published a little earlier by the even-younger E. De Giorgi in an obscure Italian journal 15, in the context of elliptic equations of type (0.9). Moreover, as commented by $\mathrm{C}$. Morrey in his MathSciNet reviews of both articles, the result of De Giorgi was simpler and seemingly stronger in that it depends only on the natural $L^{2}$ bound for $w$ rather than the $L^{\infty}$ one assumed by Nash. In fact all the main steps in [76], including the crucial moment estimate (13), do not depend on $B$ at all (the constant $B$ appears, in fact, only at the last step 35 in (31)). Thus, even though Nash's result applied, beyond the elliptic equations treated by De Giorgi, to a large class of parabolic equations, which he himself considered more fundamental 36 Nash

\footnotetext{
${ }^{34}$ In fact Nash also derives a Hölder estimate with respect to $t$.

${ }^{35} \mathrm{C}$. Villani, who has a working understanding of Nash's proof, has assured me that Nash could have easily proved the stronger result. As pointed out by C. De Lellis in his review 18, the $L^{\infty}$ bound was also later derived by Stampachia, in the context of elliptic equations.

${ }^{36}$ Here is a direct quote from his introduction: "Moreover to us, parabolic equations seem more natural than elliptic ones. It is certainly true in principle that the theory of parabolic equations includes elliptic equations as a specialization, and in applications an elliptic equation typically arises as the description of the steady state of a system which in general is described by a parabolic equation."
} 
was greatly distressed 37 by the outcome, and this may have led to the first outburst of paranoid behavior 38

De Giorgi's method was soon simplified by J. Moser in [70] by using a sequence of improving $L^{p}$ estimates, connecting $L^{2}$ to $L^{\infty}$. He then used his new iterative procedure to derive Harnack inequalities for both elliptic [71 and parabolic 72 . equations in divergence form. Similar results were later found for scalar elliptic and parabolic equations in nondivergence form by Krylov and Safanov; see [59. An excellent exposition of Nash's original proof can be found in [18.

0.2.1. Impact of the Hölder regularity paper. It is hard to overestimate the impact of the De Giorgi-Nash-Moser regularity results. These are undoubtedly the most fundamental works we have today in the general theory of elliptic and parabolic (scalar) equations, with an enormous range of applications in both physical and geometric problems.

Nash's seemingly strange idea to derive hard a priori estimates for elliptic equations in divergence form by a heat-equation approach has also paid off. It turns out that heat flows provide a powerful tool to solve difficult minimization problems. Eels and Sampson [27] were the first to use such an approach to a geometric variational problem. They were able to construct minimizers for the harmonic-map functional by using the associated gradient flow. Their successful approach inspired R. Hamilton to bypass the enormous difficulties connected to a direct variational approach for constructing (Riemannian) Einstein metric 39 by introducing his celebrated Ricci flow. As is well known, it is through the Ricci flow approach pioneered by Hamilton that G. Perelman was able to solve later the Poincaré conjecture. In his work, Perelman also made use of an entropy type quantity to derive his estimates for the nonlinear Ricci flow. The introduction of the Boltzmann entropy in the proof of a regularity result is another novel, unexpected idea in Nash's 1958 paper. For more insightful comments about this, see the beautiful and very instructive exposition of C. Villani in [19.

The hope Nash had that his new estimates, or some variation of them, would apply to solve some of the difficult problems connected with the Navier-Stokes equations failed to be realized for the simple reason that the De Giorgi-Nash-Moser techniques cannot be extended to general systems. This was first noticed by De Giorgi for the elliptic system in divergence form; see [16. In particular, one cannot hope to extend the regularity results for minimizers for convex vectorial variational problems in the spirit of Hilbert's 19th problem. Indeed, we know today that there exist nondifferentiable minimizers of smooth, uniformly convex functionals

\footnotetext{
${ }^{37} \mathrm{Nash}$, very competitive by nature, had great expectations that, together with his work on the isometric embedding, 76] would make him a compelling candidate for the Fields medal. In the end, neither De Giorgi nor Nash received the medal; obviously, one of the most inexplicable failures of a prize which is often described as the Nobel prize of mathematics. As possible consolation to the 1962 Fields committee, Nash also failed to get the Böcher prize despite his well-known frenzied attempt to withdraw his 1958 paper from Acta Mathematica in favor of the American Journal of Mathematics, in order to qualify for the prize.

${ }^{38}$ Asked about this great disappointment in Oslo in 2015, he gives a more philosophical answer: "Of course I was disappointed but one tends to find some other way to think about it. Like water building up and the lake flowing over, and then the out flow stream backing up, so it comes out another way."

${ }^{39}$ That is, Riemannian metrics verifying $R_{i j}=\lambda g_{i j}$ which can be derived from a Riemannian analogue of Einstein functional in general relativity.
} 
for domains of dimension $d \geq 3$ and targets of dimension $t \geq 2$; see 65]. Since the cases $(d \geq 1, t=1)$ and $(d=2, t \geq 1)$ are covered by De Giorgi-Nash and Morrey results, respectively, this counterexample is optimal.

Special geometric systems, however, do behave better. The best example is the work of Hamilton on Harnack inequality for the mean curvature [37] and Ricci flows [38; see also [39]. His work, which builds on the Li-Yau inequality 62 for the heat equation on a Riemannian manifold, takes maximum advantage of the maximum principle in vectorial situations where it is highly surprising that it can be still applied. Nothing similar exists for the Navier-Stokes equation or for general, realistic classes of elliptic systems occurring in continuum mechanics.

0.3. What explains Nash's unique originality? This is, of course, a question not even Nash could answer 40 Here are my own speculations, based on the little I knew of the man I have often talked with in the faculty lounge at Princeton.

(1) Choice of problems. Nothing is more important and more personal in the creative process of a mathematician than the problems one chooses to focus on. Ability to isolate the essential in a problem and concentrate relentlessly on it is a close second. Technique, lastly, is, of course, something all successful mathematicians must possess, in various degrees. It is obvious from what we know that Nash was extraordinarily deliberate in his choice of problems. In her book, S. Nassar provides a vivid portrait on how Nash took extreme precautions to make sure that a particular problem was considered important by people he respected 41 But in the end, the choices he made conformed to his own taste in problems that appear fundamental from a broad point of view, have an established pedigree, and have the potential to be transformative. He shared with both Poincar 42 and Hilbert 43 a great respect for problems connected to physics. He was interested in just about any great problem in which he thought mathematical ideas could be relevant: the foundations of quantum mechanics, general relativity, cosmology, particle physics, and, of course, also economics.

\footnotetext{
${ }^{40}$ There is this disturbing story in Nassar's book 77] according to which a Harvard colleague asked him, during a visit at at the psychiatric hospital where Nash was interned, how can he, a man of great rational power, give credence to those illogical voices he was hearing - to which Nash answered that these were the very same voices that guided him in his mathematical discoveries.

${ }^{41} \mathrm{He}$ even went so far as to probe the interest in a particular problem by, falsely, taking credit for its solution long before he actually solved it; see [77.

${ }^{42}$ In his address to the first ICM in 1897 Poincaré makes a powerful tribute for the importance of problems inspired by physics. "...The combinations that can be formed with numbers and symbols are an infinite multitude. In this thicket how shall we choose those that are worthy of our attention? Shall we be guided only by whimsy?... [This] would undoubtedly carry us far from each other, and we would rapidly cease to understand each other. But that is only the minor side of the problem. Not only will physics perhaps prevent us from getting lost, but it will also protect us from a more fearsome danger ... turning around forever in circles. History [shows that] physics has not only forced us to choose [from the multitude of problems which arise], but it has also imposed on us directions that would never have been dreamed of otherwise.... What could be more useful!"

${ }^{43}$ In [40] Hilbert emphasizes both the importance of "the outer world" motivation as well as that internal to mathematics "by means of logical combination, generalization, specialization, by separating and collecting ideas in fortunate ways". In his survey Gromov [31 attempts to give a categorical characterization of this type of internal problem. I daresay this point of view would have been quite foreign to Nash.
} 
(2) Competitiveness and independence. He was both extremely competitive and fiercely independent. As a graduate student at Princeton his learning technique was to avoid formal courses for fear of being too much influenced by a specific circumscribing point of view, and to concentrate instead on reading, preferably from the original sources, and discussions with people. He felt that being an expert in a particular subject would inhibit his originality 44 In my conversations with him, I was always struck by his ability to see any topic of discussion, even the most mundane ones, from an unusual, unexpected angle.

Despite his independence, Nash would often consult people whose judgement he trusted. This is particularly true in the case of his parabolic regularity paper in which, in addition to Nirenberg, he was heavily influenced by E. Stein, who suggested the interpolation inequality (which bears Nash's name), and L. Carlesson who had apparently initiated Nash on the use of the Boltzmann entropy. He loved talking about his ideas and was never too shy to approach anybody who could help him.

(3) Focus, persistence, and immense self-confidence. As vividly documented in Nassar's book, once Nash decided that a problem was worth his effort, he would focus on it with all his incredible intellectual powers and with a sense that nothing was impossible. It is this amazing self-confidence that allowed him to probe directions which were deemed a "no-go" by his contemporaries.

(4) Intellectual honesty. Though Nash's behavior was not always beyond reproach, he was intellectually honest to a fault and extremely demanding of himself when it came to his mathematics. I am particularly moved by the portion of his interview with Raussen and Skau where he discusses his work on the smooth embeddings and feels compelled to admit that there was a mistake in it, found 40 years after its publication by the logician R. M. Solovay. Never mind that the mistake affected only a relatively minor part of his great paper and that the mistake had been in fact corrected 20 years earlier by Gromov in 30,32, Nash spent the entire portion of the interview dedicated to this result expressing his embarrassment at finding out that he actually made a mistake.

\section{Appendix A. NASH's iteration technique}

Very roughly, Nash's method is to combine a rapidly converging scheme with an unexpected smoothing procedure meant to restore the loss of derivatives. To appreciate these ingredients, it helps to view them in the simplest abstract framework.

Recall first the simplest form of the classical implicit function theorem, i.e., the inverse function theorem, for a smooth nonlinear functional $\Phi: U \subset E \longrightarrow F$ with $U$ and open set in $E$, and $E, F$ Banach spaces.

Proposition A.1. A $C^{1}$ functional $\Phi: U \subset E \longrightarrow F$ is locally invertibl 45 near $u_{0} \in U$ if its Frechet derivative $\Phi^{\prime}(u): E \longrightarrow F$ is an invertible linear map at $u_{0}$.

\footnotetext{
${ }^{44}$ In this he was heavily influenced by the example of Einstein.

45 i.e., for any $f$ in a neighborhood of $f_{0}=\Phi\left(u_{0}\right)$, the equation $\Phi(u)=f$ admits a unique solution $u$ near $u_{0}$.
} 
The proof can be easily derived by the recursion formula

$$
u_{i+1}=u_{i}+\Phi^{\prime}\left(u_{0}\right)^{-1}\left(f-\Phi\left(u_{i}\right)\right), \quad i=0,1 \ldots
$$

Note that the scheme, which depends only on the invertibility properties of the linear operator $\Phi^{\prime}\left(u_{0}\right)$, is linearly convergent with respect to the error term $e_{i}=$ $\Phi\left(u_{i}\right)-f$, provided that $f$ is sufficiently close to $f_{0}$.

This simple iteration method does not work well for quasilinear or fully nonlinear, nonelliptic, partial differential equations, such as the Burger equation

$$
\partial_{t} u+u \partial_{x} u=f, \quad u(0, x)=0 .
$$

In this case one uses instead Newton's quadratic iteration scheme

$$
u_{i+1}=u_{i}+\Phi^{\prime}\left(u_{i}\right)^{-1}\left(f-\Phi\left(u_{i}\right)\right),
$$

which requires however that $\Phi^{\prime}(u)$ be invertible not only at $u_{0}$ but in a full neighborhood of it. This is the method of choice for treating general quasilinear systems of hyperbolic laws.

In Nash's isometric embedding problem, however, this iteration still leads to a loss of two derivatives. To properly state Nash's hard implicit function theorem, one has to leave the simple functional case of Banach spaces, and place oneself in the context of indexed families 46 of Banach spaces $\left(E_{a}\right)_{a \geq 0},\left(F_{a}\right)_{a \geq 0}$ such that both verify $E_{b} \subset E_{a}$ and ||$_{a} \leq||_{b}$ for $a \leq b$.

Assume also given a family of smoothing operators

$$
S_{\theta}: E_{0} \longrightarrow E_{\infty}:=\bigcap_{a \geq 0} E_{a}
$$

verifying the properties

$$
\begin{aligned}
\left|S_{\theta} u\right|_{b} & \lesssim \theta^{b-a}|u|_{a}, & & \text { fror } \quad b \leq a, \\
\left|\left(I-S_{\theta}\right) u\right|_{b} & \lesssim \theta^{b-a}|u|_{a}, & & \text { fror } \quad b>a .
\end{aligned}
$$

Assume a similar family of smoothing operators acting on the spaces $F_{a}$.

Assume, given a nonlinear map, $\Phi: V_{\lambda} \cap E_{\tilde{a}} \longrightarrow F_{\lambda}$ with $\tilde{a}>2 \lambda$ such that $\Phi(0)=0$ and $V_{\lambda}:=\left\{u \in E_{\lambda} /|u|_{\lambda} \leq 1\right\}$.

Assume in addition that $\Phi$ verifies the following conditions:

(1) $\Phi$ is twice differentiable, and the bilinear map $\Phi^{\prime \prime}(u)$ verifies

$$
\left|\left(\Phi^{\prime \prime}(u) v, w\right)\right|_{a} \lesssim|v|_{a}|w|_{0}+|w|_{0}|w|_{a}+|u|_{a}|v|_{0}|w|_{0}
$$

for any $u \in E_{\infty} \cap V_{\lambda}, v, w \in E_{\infty}$, and any $a \in[0, \tilde{a}]$.

(2) For all $u \in V_{\lambda} \cap E_{\infty}$, the linear map $L=\Phi^{\prime}(u)$ has a right inverse $L^{-1}(u)$ : $F_{\infty} \longrightarrow E_{\tilde{a}}$ verifying the tame estimate, for all $u \in V_{\lambda} \cap E_{\infty}$ and any $g \in F_{\infty}$,

$$
\left|L^{-1}(u) g\right|_{a} \lesssim|g|_{a+\lambda}+|g|_{\lambda}|u|_{a+\lambda} .
$$

These are enough to state the following,

Theorem (Nash, Moser, and Hörmander). For any $f \in F_{2 \beta}$, where $\beta>\lambda$, such that $|f|_{2 \beta} \leq \delta$, sufficiently small, there exist a sequence $u_{i} \in V_{\lambda} \cap E_{\tilde{a}}$ such that $u_{i} \longrightarrow u$ in $E_{\lambda}$ and $\Phi\left(u_{i}\right) \longrightarrow f$ in $F_{a}$, for any $a<2 \beta$.

\footnotetext{
${ }^{46}$ Fréchet spaces would be natural but a bit cumbersome to explain.
} 
The proof of the theorem is based on an iteration scheme combined with a smoothing procedure. The simplest such procedure, introduced by Moser, has the form,

$$
u_{i+1}=u_{i}+S_{i} L^{-1}\left(u_{i}\right)\left(f-\Phi\left(u_{i}\right)\right), \quad S_{i}:=S_{\theta_{i}}, \quad \theta_{i}=2^{\alpha^{i}},
$$

for some $1<\alpha<2$. Nash used instead a continuous convergence procedure. Hörmander introduced yet another scheme which is, in fact, a discretized version of Nash's original scheme,

$$
u_{i+1}=u_{i}+\left(\Phi^{\prime}\left(S_{i} u_{i}\right)\right)^{-1} g_{i}, \quad S_{i}=2^{i},
$$

where the $g_{i}$ 's are defined in such a way that the sequence $u_{i}$ converges formally to a solution of $\Phi(u)=f$. Introducing $L_{i}:=\Phi^{\prime}\left(S_{i} u_{i}\right), L_{i}^{-1}$ its right inverse, and $\dot{u}_{i}:=u_{i+1}-u_{i}$,

$$
\dot{u}_{i}=L_{i}^{-1} g_{i}
$$

we choose the sequence $g_{i}$ according to the following procedure.

By a Taylor expansion around $u_{i}$ and denoting by $e_{i}^{\prime \prime}$ the quadratic terms in $\left(u_{i+1}-u_{i}\right)$, i.e.,

$$
e_{i}^{\prime \prime}=\Phi\left(u_{i+1}\right)-\Phi\left(u_{i}\right)-\Phi^{\prime}\left(u_{i}\right)\left(u_{i+1}-u_{i}\right),
$$

we deduce, using (A.8),

$$
\begin{aligned}
\Phi\left(u_{i+1}\right)-\Phi\left(u_{i}\right) & =\Phi^{\prime}\left(u_{i}\right)\left(u_{i+1}-u_{i}\right)+e_{i}^{\prime \prime} \\
& =\Phi^{\prime}\left(u_{i}\right) L_{i} g_{i}+e_{i}^{\prime \prime} \\
& =\left(\Phi^{\prime}\left(u_{i}\right)-L_{i}\right) L_{i} g_{i}+g_{i}+e_{i}^{\prime \prime} .
\end{aligned}
$$

Hence,

$$
\begin{aligned}
\Phi\left(u_{i+1}\right)-\Phi\left(u_{i}\right) & =g_{i}+e_{i} \\
e_{i}: & =e_{i}^{\prime}+e_{i}^{\prime \prime} \\
e_{i}^{\prime}: & =\left(\Phi^{\prime}\left(u_{i}\right)-L_{i}\right) \dot{u}_{i} \\
e_{i}^{\prime \prime}: & =\Phi\left(u_{i+1}\right)-\Phi\left(u_{i}\right)-\Phi^{\prime}\left(u_{i}\right) \dot{u}_{i} .
\end{aligned}
$$

Adding, we deduce

$$
\Phi\left(u_{i+1}\right)=\sum_{j=0}^{i} g_{j}+E_{i}+e_{i}=\sum_{j=0}^{i} g_{j}+S_{i} E_{i}+e_{i}+\left(1-S_{i}\right) E_{i},
$$

where $E_{i}=\sum_{j=0}^{i-1} e_{i}$. To ensure that $\Phi\left(u_{i+1}\right) \rightarrow f$, we choose the sequence $g_{i}$ such that

$$
\sum_{j=0}^{i} g_{j}+S_{i} E_{i}=S_{i} f
$$

Thus, recursively,

$$
\begin{aligned}
g_{0} & =S_{0}(f), \\
g_{i} & =\left(S_{i}-S_{i-1}\right)\left(f-E_{i-1}\right),
\end{aligned}
$$

and

$$
\Phi\left(u_{i}\right)=S_{i} f+e_{i}+\left(I-S_{i}\right) E_{i} .
$$


Thus, given the previous steps $u_{0}, u_{1}, \ldots, u_{i}$, we can determine the sequence $\dot{u}_{0}, \dot{u}_{1}, \ldots, \dot{u}_{i-1}$ which by (A.9) determines the sequence $e_{0}, \ldots, e_{i-1}$. Using (A.10), we can then determine the sequence $g_{i}$. The new iterate $u_{i+1}$ is then given according to A.7.

The proof of the theorem is done by systematically estimating the error terms, using its underlying assumptions and the properties of the smoothing operators; see [43] or [52] for a short presentation.

\section{ACKNOWLEDGMENTS}

I warmly thank P. Isett and V. Vicol for their invaluable help in connection with my presentation of the recent developments concerning the Onsager conjecture.

\section{About the Author}

Sergiu Klainerman is professor of mathematics at Princeton University. He works on partial differential equations with a special emphasis on general relativity and geometric nonlinear wave equations.

\section{REFERENCES}

[1] S. Alinhac, Blowup for nonlinear hyperbolic equations, Progress in Nonlinear Differential Equations and their Applications, 17, Birkhäuser Boston, Inc., Boston, MA, 1995. MR.1339762

[2] C. W. Bardos and E. S. Titi, Mathematics and turbulence: where do we stand?, J. Turbul. 14 (2013), no. 3, 42-76, DOI 10.1080/14685248.2013.771838. MR 3174319

[3] Y. Fourès-Bruhat, Théorème d'existence pour certains systèmes d'équations aux dérivées partielles non linéaires (French), Acta Math. 88 (1952), 141-225. MR0053338

[4] J. Bourgain and D. Li, Strong ill-posedness of the incompressible Euler equation in borderline Sobolev spaces, Invent. Math. 201 (2015), no. 1, 97-157, DOI 10.1007/s00222-014-0548-6. MR 3359050

[5] T. Buckmaster, C. De Lellis, P. Isett, and L. Székelyhidi Jr., Anomalous dissipation for $\frac{1}{5}$-Hölder Euler flows, Preprint, 2014. To appear in Ann. of Math.

[6] T. Buckmaster, C. De Lellis, L. Székelyhidi. Dissipative Euler flows with Onsager critical spatial regularity. Commun. Pure Appl. Math. 69 (2016), no. 9, 1613-1670.

[7] T. Buckmaster, N. Masmoudi, and V. Vicol. Onsager's conjecture and Kolmogorov's 5/3 law. Preprint (2016).

[8] E. Cartan, Sur la possibilité de plonger un espace riemannien donné dans un espace euclidien, Ann. Soc. Polon. Math. 6 (1927), 1-7.

[9] P. Constantin, W. E, and E. S. Titi, Onsager's conjecture on the energy conservation for solutions of Euler's equation, Comm. Math. Phys. 165 (1994), no. 1, 207-209. MR.1298949

[10] D. Cordoba, D. Faraco, and F. Gancedo, Lack of uniqueness for weak solutions of the incompressible porous media equation, Arch. Ration. Mech. Anal. 200 (2011), no. 3, 725-746, DOI 10.1007/s00205-010-0365-z. MR2796131

[11] A. Cheskidov, P. Constantin, S. Friedlander, and R. Shvydkoy, Energy conservation and Onsager's conjecture for the Euler equations, Nonlinearity 21 (2008), no. 6, 1233-1252, DOI 10.1088/0951-7715/21/6/005. MR2422377

[12] E. Chiodaroli, A counterexample to well-posedness of entropy solutions to the compressible Euler system, J. Hyperbolic Differ. Equ. 11 (2014), no. 3, 493-519, DOI 10.1142/S0219891614500143. MR3261301

[13] C. Mouhot and C. Villani, On Landau damping, Acta Math. 207 (2011), no. 1, 29-201, DOI 10.1007/s11511-011-0068-9. MR.2863910

[14] S. Conti, C. De Lellis, and L. Székelyhidi Jr., h-principle and rigidity for $C^{1, \alpha}$ isometric embeddings, Nonlinear partial differential equations, Abel Symp., vol. 7, Springer, Heidelberg, 2012, pp. 83-116, DOI 10.1007/978-3-642-25361-4_5. MR3289360 
[15] E. De Giorgi, Sulla differenziabilità e l'analiticità delle estremali degli integrali multipli regolari (Italian), Mem. Accad. Sci. Torino. Cl. Sci. Fis. Mat. Nat. (3) 3 (1957), 25-43. MR.0093649

[16] E. De Giorgi, Un esempio di estremali discontinue per un problema variazionale di tipo ellittico (Italian), Boll. Un. Mat. Ital. (4) 1 (1968), 135-137. MR0227827

[17] D. M. DeTurck, Deforming metrics in the direction of their Ricci tensors, J. Differential Geom. 18 (1983), no. 1, 157-162. MR697987

[18] C. De Lellis The masterpieces of John Forbes Nash Jr., to appear as a contribution to an Abel symposium

[19] C. De Lellis, contributing editor, John Forbes Nash Jr. (1928-2015), Notices Amer. Math. Soc. 63 (2016), no. 5, 492-501.

[20] C. De Lellis and L. Székelyhidi Jr., The h-principle and the equations of fluid dynamics, Bull. Amer. Math. Soc. (N.S.) 49 (2012), no. 3, 347-375, DOI 10.1090/S0273-0979-2012-01376-9. MR2917063

[21] C. De Lellis and L. Székelyhidi Jr., The Euler equations as a differential inclusion, Ann. of Math. (2) 170 (2009), no. 3, 1417-1436, DOI 10.4007/annals.2009.170.1417. MR2600877

[22] C. De Lellis and L. Székelyhidi Jr., Dissipative continuous Euler flows, Invent. Math. 193 (2013), no. 2, 377-407, DOI 10.1007/s00222-012-0429-9. MR.3090182

[23] C. De Lellis and L. Székelyhidi Jr., Dissipative Euler flows and Onsager's conjecture, J. Eur. Math. Soc. (JEMS) 16 (2014), no. 7, 1467-1505, DOI 10.4171/JEMS/466. MR3254331

[24] C. De Lellis and L. Székelyhidi Jr., On admissibility criteria for weak solutions of the Euler equations, Arch. Ration. Mech. Anal. 195 (2010), no. 1, 225-260, DOI 10.1007/s00205-0080201-x. MR 2564474

[25] C. DeLellis, D. Ianuen, and L.Székelyhidi, A Nash-Kuiper theorem for $C^{1,1 / 5-\delta}$ immersions of surfaces in 3 dimensions, arXiv:1510:01934v1, Oct. 2015.

[26] S. Daneri and L. Szekelyhidi, Jr., Non-uniqueness and h-principle for Hölder-continuous weak solutions of the Euler equations, Preprint. 2016.

[27] J. Eells Jr. and J. H. Sampson, Harmonic mappings of Riemannian manifolds, Amer. J. Math. 86 (1964), 109-160. MR0164306

[28] G. L. Eyink, Energy dissipation without viscosity in ideal hydrodynamics. I. Fourier analysis and local energy transfer, Phys. D 78 (1994), no. 3-4, 222-240, DOI 10.1016/01672789(94)90117-1. MR1302409

[29] G. L. Eyink and K. R. Sreenivasan, Onsager and the theory of hydrodynamic turbulence, Rev. Modern Phys. 78 (2006), no. 1, 87-135, DOI 10.1103/RevModPhys.78.87. MR2214822

[30] M. L. Gromov, Isometric imbeddings and immersions (Russian), Dokl. Akad. Nauk SSSR 192 (1970), 1206-1209. MR0275456

[31] M. Gromov, Geometric, algebraic, and analytic descendants of Nash isometric embedding theorems, Bull. Amer. Math. Soc. 54 (2016), no. 2, to appear.

[32] M. Gromov, Partial differential relations, Ergebnisse der Mathematik und ihrer Grenzgebiete (3) [Results in Mathematics and Related Areas (3)], vol. 9, Springer-Verlag, Berlin, 1986. MR864505

[33] M. Günther, Zum Einbettungssatz von J. Nash (German), Math. Nachr. 144 (1989), 165-187, DOI 10.1002/mana.19891440113. MR1037168

[34] M. Günther, Isometric embeddings of Riemannian manifolds, Proceedings of the International Congress of Mathematicians, Vol. I, II (Kyoto, 1990), Math. Soc. Japan, Tokyo, 1991, pp. 1137-1143. MR 1159298

[35] R. S. Hamilton, The inverse function theorem of Nash and Moser, Bull. Amer. Math. Soc. (N.S.) 7 (1982), no. 1, 65-222, DOI 10.1090/S0273-0979-1982-15004-2. MR656198

[36] R. S. Hamilton, Three-manifolds with positive Ricci curvature, J. Differential Geom. 17 (1982), no. 2, 255-306. MR664497

[37] R. S. Hamilton, Harnack estimate for the mean curvature flow, J. Differential Geom. 41 (1995), no. 1, 215-226. MR 1316556

[38] R. S. Hamilton, The Harnack estimate for the Ricci flow, J. Differential Geom. 37 (1993), no. 1, 225-243. MR.1198607

[39] R. S. Hamilton, A matrix Harnack estimate for the heat equation, Comm. Anal. Geom. 1 (1993), no. 1, 113-126, DOI 10.4310/CAG.1993.v1.n1.a6. MR.1230276 
[40] D. Hilbert, Mathematical problems, Bull. Amer. Math. Soc. (N.S.) 37 (2000), no. 4, 407-436 (electronic), DOI 10.1090/S0273-0979-00-00881-8. Reprinted from Bull. Amer. Math. Soc. 8 (1902), 437-479. MR1779412

[41] P. Hintz and A. Vasy, The global nonlinear stability of the Kerr-De Sitter family of black holes, arXiv:1606.04014v1

[42] L. Hörmander, The boundary problems of physical geodesy, Arch. Rational Mech. Anal. 62 (1976), no. 1, 1-52. MR0602181

[43] L. Hörmander, Implicit function theorems, lecture notes, Stanford Univ., 1977.

[44] P. Isett, Hölder continuous Euler flows in three dimensions with compact support in time, Preprint. 2012.

[45] P. Isett, A proof of Onsager's conjecture, preprint Aug. 2016, arXiv:1608.08301 [math.AP], 13 Jun 2016

[46] P. Isett and V. Vicol, Hölder continuous solutions of active scalar equations, Ann. PDE 1 (2015), no. 1, Art. 2, 77. MR3479065

[47] H. Jacobowitz, Implicit function theorems and isometric embeddings, Ann. of Math. (2) 95 (1972), 191-225. MR0307127

[48] M. Janet, Sur la possibilité de plonger un espace riemannien donné dans un espace euclidien. Ann. Soc. Polon. Math. 5 (1926), 38-43.

[49] S. Klainerman, Global existence for nonlinear wave equations, Comm. Pure Appl. Math. 33 (1980), no. 1, 43-101, DOI 10.1002/cpa.3160330104. MR.544044

[50] S. Klainerman, Uniform decay estimates and the Lorentz invariance of the classical wave equation, Comm. Pure Appl. Math. 38 (1985), no. 3, 321-332, DOI 10.1002/cpa.3160380305. MR.784477

[51] S. Klainerman, PDE as a unified subject, Geom. Funct. Anal. Special Volume (2000), 279-315, DOI 10.1007/978-3-0346-0422-2_10. GAFA 2000 (Tel Aviv, 1999). MR1826256

[52] S. Klainerman, Notes on Nash-Moser-Hörmander scheme, unpublished manuscript.

[53] S. Klainerman and A. Majda, Compressible and incompressible fluids, Comm. Pure Appl. Math. 35 (1982), no. 5, 629-651, DOI 10.1002/cpa.3160350503. MR668409

[54] S. Klainerman and G. Ponce, Global, small amplitude solutions to nonlinear evolution equations, Comm. Pure Appl. Math. 36 (1983), no. 1, 133-141, DOI 10.1002/cpa.3160360106. MR680085

[55] S. Klainerman, I. Rodnianski, and J. Szeftel, The bounded $L^{2}$ curvature conjecture, Invent. Math. 202 (2015), no. 1, 91-216, DOI 10.1007/s00222-014-0567-3. MR3402797

[56] A. Kolmogoroff, The local structure of turbulence in incompressible viscous fluid for very large Reynold's numbers, C. R. (Doklady) Acad. Sci. URSS (N.S.) 30 (1941), 301-305. MR0004146

[57] J. Krieger and D. Tataru, Global well-posedness for the Yang-Mills equation in $4+1$ dimensions. Small energy. To appear in Annals of Math.

[58] J. Krieger and W. Schlag, Concentration compactness for critical wave maps, EMS Monographs in Mathematics, European Mathematical Society (EMS), Zürich, 2012. MR2895939

[59] N. V. Krylov and M. V. Safonov, A property of the solutions of parabolic equations with measurable coefficients (Russian), Izv. Akad. Nauk SSSR Ser. Mat. 44 (1980), no. 1, 161175, 239. MR 563790

[60] N. H. Kuiper, On $C^{1}$-isometric imbeddings. I, II, Nederl. Akad. Wetensch. Proc. Ser. A. 58 = Indag. Math. 17 (1955), 545-556, 683-689. MR0075640

[61] M. Kuranishi, Application of $\bar{\partial}_{b}$ to deformation of isolated singularities, Several complex variables (Proc. Sympos. Pure Math., Vol. XXX, Part 1, Williams Coll., Williamstown, Mass., 1975), Amer. Math. Soc., Providence, R.I., 1977, pp. 97-106. MR0454072

[62] P. Li and S.-T. Yau, On the parabolic kernel of the Schrödinger operator, Acta Math. 156 (1986), no. 3-4, 153-201, DOI 10.1007/BF02399203. MR834612

[63] H. Lindblad, Well-posedness for the motion of an incompressible liquid with free surface boundary, Ann. of Math. (2) 162 (2005), no. 1, 109-194, DOI 10.4007/annals.2005.162.109. MR2178961

[64] T. Luo, C. Xie, and Z. Xin, Non-uniqueness of admissible weak solutions to compressible Euler systems with source terms, Adv. Math. 291 (2016), 542-583, DOI 10.1016/j.aim.2015.12.027. MR.3459023

[65] C. Mooney and O. Savin, Some singular minimizers in low dimensions in the calculus of variations, Arch. Ration. Mech. Anal. 221 (2016), no. 1, 1-22, DOI 10.1007/s00205-0150955-x. MR3483890 
[66] C. B. Morrey Jr., On the solutions of quasi-linear elliptic partial differential equations, Trans. Amer. Math. Soc. 43 (1938), no. 1, 126-166, DOI 10.2307/1989904. MR.1501936

[67] C. B. Morrey Jr., Second-order elliptic systems of differential equations, Contributions to the theory of partial differential equations, Annals of Mathematics Studies, no. 33, Princeton University Press, Princeton, N. J., 1954, pp. 101-159. MR0068091

[68] J. Moser, A new technique for the construction of solutions of nonlinear differential equations, Proc. Nat. Acad. Sci. U.S.A. 47 (1961), 1824-1831. MR0132859

[69] J. Moser, A rapidly convergent iteration method and non-linear partial differential equations. I, Ann. Scuola Norm. Sup. Pisa (3) 20 (1966), 265-315. MR0199523

[70] J. Moser, A new proof of De Giorgi's theorem concerning the regularity problem for elliptic differential equations, Comm. Pure Appl. Math. 13 (1960), 457-468. MR0170091

[71] J. Moser, On Harnack's theorem for elliptic differential equations, Comm. Pure Appl. Math. 14 (1961), 577-591. MR0159138

[72] J. Moser, A Harnack inequality for parabolic differential equations, Comm. Pure Appl. Math. 17 (1964), 101-134. MR.0159139

[73] S. Müller and V. Šverák, Convex integration for Lipschitz mappings and counterexamples to regularity, Ann. of Math. (2) 157 (2003), no. 3, 715-742, DOI 10.4007/annals.2003.157.715. MR.1983780

[74] J. Nash, $C^{1}$ isometric imbeddings, Ann. of Math. (2) 60 (1954), 383-396. MR0065993

[75] J. Nash, The imbedding problem for Riemannian manifolds, Ann. of Math. (2) 63 (1956), 20-63. MR.0075639

[76] J. Nash, Continuity of solutions of parabolic and elliptic equations, Amer. J. Math. 80 (1958), 931-954. MR0100158

[77] S. Nassar, A beautiful mind: A biography of John Forbes Nash, Jr., winner of the Nobel Prize in economics, Simon \& Schuster, New York, 1994. ISBN 0-684-81906-6

[78] L. Nirenberg, The Weyl and Minkowski problems in differential geometry in the large, Comm. Pure Appl. Math. 6 (1953), 337-394. MR0058265

[79] L. Nirenberg, An abstract form of the nonlinear Cauchy-Kowalewski theorem, J. Differential Geometry 6 (1972), 561-576. Collection of articles dedicated to S. S. Chern and D. C. Spencer on their sixtieth birthdays. MR0322321

[80] S.-J. Oh and D. Tataru, Local well-posedness of the $(4+1)$-dimensional Maxwell-KleinGordon equation at energy regularity, Ann. PDE 2 (2016), no. 1, Art. 2, 70, DOI 10.1007/s40818-016-0006-4. MR 3462105

[81] S.-J. Oh and D. Tataru, Local well-posedness of the $(4+1)$-dimensional Maxwell-KleinGordon equation at energy regularity, Ann. PDE 2 (2016), no. 1, Art. 2, 70, DOI 10.1007/s40818-016-0006-4. MR3462105

[82] L. Onsager, Statistical hydrodynamics, Nuovo Cimento (9) 6 (1949), no. Supplemento, 2 (Convegno Internazionale di Meccanica Statistica), 279-287. MR.0036116

[83] D. G. Schaeffer, A stability theorem for the obstacle problem, Advances in Math. 17 (1975), no. 1, 34-47. MR0380093

[84] V. Scheffer, An inviscid flow with compact support in space-time, J. Geom. Anal. 3 (1993), no. 4, 343-401, DOI 10.1007/BF02921318. MR1231007

[85] J. Schwartz, On Nash's implicit functional theorem, Comm. Pure Appl. Math. 13 (1960), 509-530. MR0114144

[86] F. Sergeraert, Un théorème de fonctions implicites sur certains espaces de Fréchet et quelques applications (French), Ann. Sci. École Norm. Sup. (4) 5 (1972), 599-660. MR0418140

[87] A. Shnirelman, On the nonuniqueness of weak solution of the Euler equation, Comm. Pure Appl. Math. 50 (1997), no. 12, 1261-1286, DOI 10.1002/(SICI)10970312(199712)50:12〈1261::AID-CPA3〉3.3.CO;2-4. MR.1476315

[88] A. Shnirelman, Weak solutions with decreasing energy of incompressible Euler equations, Comm. Math. Phys. 210 (2000), no. 3, 541-603, DOI 10.1007/s002200050791. MR1777341

[89] J. Shatah, Global existence of small solutions to nonlinear evolution equations, J. Differential Equations 46 (1982), no. 3, 409-425, DOI 10.1016/0022-0396(82)90102-4. MR681231

[90] J. Sterbenz and D. Tataru, Energy dispersed large data wave maps in $2+1$ dimensions, Comm. Math. Phys. 298 (2010), no. 1, 139-230, DOI 10.1007/s00220-010-1061-4. MR2657817

[91] J. Sterbenz and D. Tataru, Regularity of wave-maps in dimension $2+1$, Comm. Math. Phys. 298 (2010), no. 1, 231-264, DOI 10.1007/s00220-010-1062-3. MR2657818 
[92] R. Shvydkoy, Lectures on the Onsager conjecture, Discrete Contin. Dyn. Syst. Ser. S 3 (2010), no. 3, 473-496, DOI 10.3934/dcdss.2010.3.473. MR2660721

[93] L. Székelyhidi Jr., Relaxation of the incompressible porous media equation (English, with English and French summaries), Ann. Sci. Éc. Norm. Supér. (4) 45 (2012), no. 3, 491-509. MR.3014484

[94] T. Tao, Global regularity of wave maps. II. Small energy in two dimensions, Comm. Math. Phys. 224 (2001), no. 2, 443-544, DOI 10.1007/PL00005588. MR.1869874

[95] T. Tao, Global Regularity of Wave Maps III-VII, preprints.

[96] H. Weyl, Ueber die Bestimmung einer geschlossenen konvexen Fläche durch ihr Linienelement Vierteljahrschrift Naturforsch. Gesell. Zurich, $3: 2$ (1916) 40-72.

Princeton University

E-mail address: seri@math.princeton.edu 